\title{
Contributions of functional Magnetic Resonance Imaging (fMRI) to sport concussion evaluation
}

\author{
Alain Ptito $^{\mathrm{a}, *}$, Jen-Kai Chen ${ }^{\mathrm{a}}$ and Karen M. Johnston ${ }^{\mathrm{b}}$ \\ ${ }^{a}$ Montreal Neurological Institute McGill University, Montreal, Quebec, Canada \\ ${ }^{\mathrm{b}}$ Concussion Clinic NeuroRehabilitation Program, Toronto Rehabilitation Division of Neurosurgery, University of \\ Toronto, Toronto, Ontario, Canada
}

\begin{abstract}
Mild traumatic brain injury (mTBI) in contact sport is a problem of such magnitude that improved approaches to diagnosis, investigation and management are urgent. Concussion has traditionally been described as a transient, fully reversible, cerebral dysfunction. However, this seemingly 'mild' injury sometimes results in long-lasting and disabling post-concussion symptoms (PCS) and abnormal neuropsychological profiles characteristic of frontal and/or temporal lobe dysfunction. At present, the pathological changes following concussion remain unclear, but it is now widely accepted that concussion results mainly in functional disturbance rather than structural damage. Therefore, functional imaging techniques can help in demonstrating brain abnormalities undetectable by structural imaging methods.

This paper will review the use of functional magnetic resonance imaging (fMRI) in studies of concussion. Our existing and ongoing fMRI studies will be described as examples to highlight the potential and contribution of this non-invasive functional neuroimaging technique in the assessment of sports-related concussion and its management.
\end{abstract}

Keywords: Concussion, functional Magnetic Resonance Imaging (fMRI), post-concussive symptoms, depression

\section{Introduction}

In 1998, the NIH Consensus Statement [44] indicated that head trauma is common and results mainly from motor vehicle accidents, acts of violence, and sports injuries, and is more than twice as frequent in males as in females. Because the neuropathological, neurological and neurobehavioral consequences of mTBI and concussion overlap, these two terms will be used interchangeably here. Concussion has recently been redefined with new emphasis on functional rather than structural injury, transient nature, and a graded set of clinical syndromes that may or may not involve loss of

*Address for correspondence: Alain Ptito, PhD, Neuropsychology Department/Cognitive Neuroscience Unit, Montreal Neurological Institute, 3801 University Street, Montreal, Quebec, Canada H3A 2B4. Tel.: +1 514398 8906; Fax: +1 514398 1338; E-mail: alain. ptito@mcgill.ca. consciousness [2-4]. This is because mTBI can produce demonstrable structural brain damage whether or not a loss of consciousness occurs at the time of injury [54]. The recent Summary and Agreement Statement of the $2^{\text {nd }}$ International Conference on Concussion in Sport (Prague, 2004) [43] did not change the definition beyond noting that, in some cases post concussive symptoms (PCS) may be prolonged or persistent and that concussion severity could only be determined in retrospect after all concussion symptoms have cleared, the neurological examination is normal and cognitive function has returned to baseline [5]. It did, however, come to an understanding that concussion may be categorized for management purposes as either simple (symptoms resolving without complication over 7-10 days) or complex (persistent symptom recurrence with exertion, convulsions, prolonged loss of consciousness, prolonged cognitive impairment). 
Concussion used to be considered as a temporary fluctuation in consciousness with no long-term effect on cognition. As such, no significant damage to neurons was believed to have ensued. Recent popular cases of athletes and other individuals with persistent cognitive deficits following concussion (the post-concussion syndrome) suggest that this is not always the case [6]. Concussion is a particular concern in contact sports, such as hockey and football, because once cleared to play, the players return to an environment in which a head injury has a reasonable likelihood of recurring. In North America, there is a growing list of athletes whose careers have been jeopardized or ended prematurely due to the effects of multiple concussions. An emerging hypothesis in the study of neurotrauma is that repeated mTBI may cause cumulative damage to the brain [19,51], which could ultimately result in memory and learning dysfunction. Recurrent brain injuries are common in many sports [30] as some include blows to the head (contact sports) or use the head as part of playing the game (e.g. heading the ball in soccer). Studies have shown that soccer players who regularly head the ball have more brain concussions, and show impairment on multiple neuropsychological tests $[37,39]$ but see [22]. In American football, players with repeated concussions perform worse on neuropsychological tests than those with either a single concussion or no history of concussion [13]. Many concussions result in deterioration of planning and memory [38], functions to which the frontal and temporal lobes (particularly the hippocampus), respectively, contribute. After a concussion, individuals often exhibit diverse symptomatology and abnormal neuropsychological profiles involving learning and memory of new material, presumed to be associated with hippocampal function, as well as working memory, attention, information processing speed, and integrative tasks that contribute to executive function (frontal-lobe function).

\subsection{Functional MRI in concussion}

Whereas imaging techniques such as CT and MRI can furnish knowledge of intracranial anatomical abnormalities, functional techniques such as fMRI could provide insights into the pathophysiological and functional sequelae of injury. Although information on brain function has traditionally been obtained using radioactive tracer techniques to measure changes in cerebral blood flow in response to functional activations, fMRI has become the method of choice for making brain-behavior inferences. FMRI detects the conse- quence of neuronal activation in cortical matter by observing regional changes in either $\mathrm{T} 1$ from increased blood perfusion, or T2 from reduced paramagnetic deoxyhemoglobin levels. The most common approach is based on blood oxygenation changes, and the signal contrast thus generated has been termed BOLD, for blood oxygenation-level dependent [50]. FMRI studies hold great potential for widespread research and clinical use because fMRI does not require exposure to any radioactive substance, has temporal resolution limited only by brain hemodynamics, and spatial resolution comparable to that of conventional MRI. Thus, fMRI offers us the opportunity to use within as well as across subjects designs. This promotes the implementation of prospective, neurophysiologic investigations in which preinjury baseline measures of neural function are obtained for each participant in anticipation of future deleterious events, such as brain injury.

In fact, fMRI has been used with concussed patients in a prospective study that used an auditory $n$-back working memory task with a high processing load [40]. Individuals with mTBI showed an increase in activation only in right frontal and parietal cortex while controls showed a greater increase in activation in parietal cortical areas bilaterally. Also, mTBI subjects had smaller increases in brain activation than controls. The authors have used fMRI to probe episodic memory in mTBI. Subjects learned a 10 -item word list and passively listened to the learned words interspersed with 38 novel words during fMRI acquisition in an event-related design. In healthy controls, familiar words were associated with predominant activation of right prefrontal cortex and novel words with medial temporal cortical activation. Significant reductions in activation were observed in the mTBI group suggesting that within one month of injury, there are demonstrable differences in the ability to activate, allocate, and modulate memory processing resources [41]. Jantzen et al. [25] carried out fMRI and neuropsychological testing in four athletes before and after concussion. FMRI after injury showed increased activation in the parietal and lateral frontal and cerebellar regions when compared with preinjury, in the absence of changes in cognitive performance.

In an fMRI study carried out in our laboratory, Chen et al. [12] compared brain activations of 16 concussed athletes with a group of normal controls using the externally ordered task, one of the working memory tasks devised by Petrides and validated in studies of patients with lateral frontal lesions, monkeys with dorsolateral prefrontal cortex (DLPC) lesions and functional neuroimaging work with PET and fMRI [45-49]. The 
a)

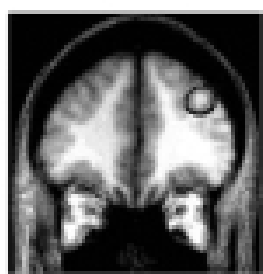

Control Group

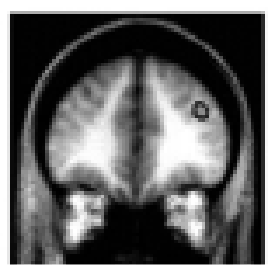

Athlete Group b)

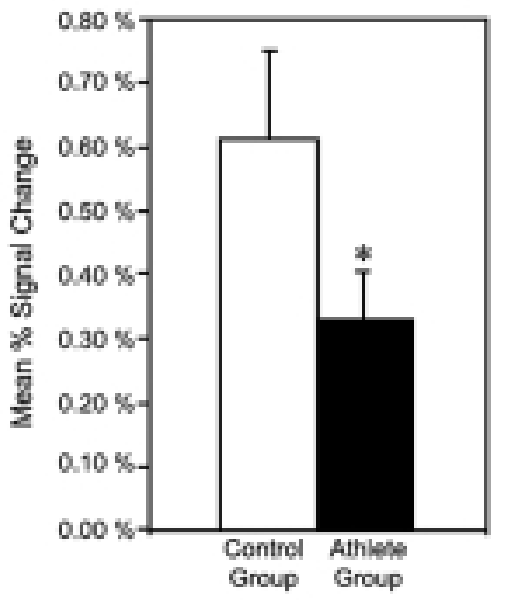

c)
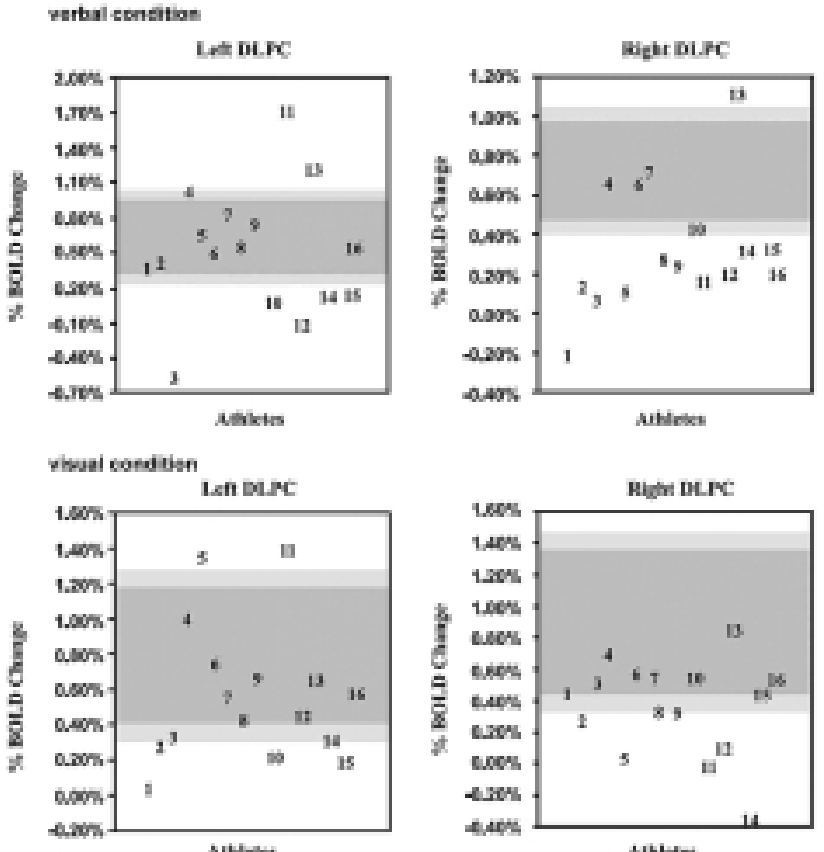

Fig. 1. a) Average t-statistic maps comparing control and concussed groups for working memory tasks; note reduced activation for the concussed group; b) Reduced percent BOLD signal change for athletes; c) Summary of the \% signal change in the mid-DLPC for each athlete while performing the working memory task. The dark and light shaded areas represent $90 \%$ and $95 \%$ quantiles of the control group, respectively. Adapted from [21].

results showed the expected fronto-cortical activations for eight normal control subjects (NC). All the athletes but one had subjective complaints of post concussive symptoms (PCS) at the time of the functional imaging study. Interestingly, the one symptom-free athlete was also the only one showing an activation pattern similar to that of the control group, while all the symptomatic athletes displayed a different activation pattern, regard- 

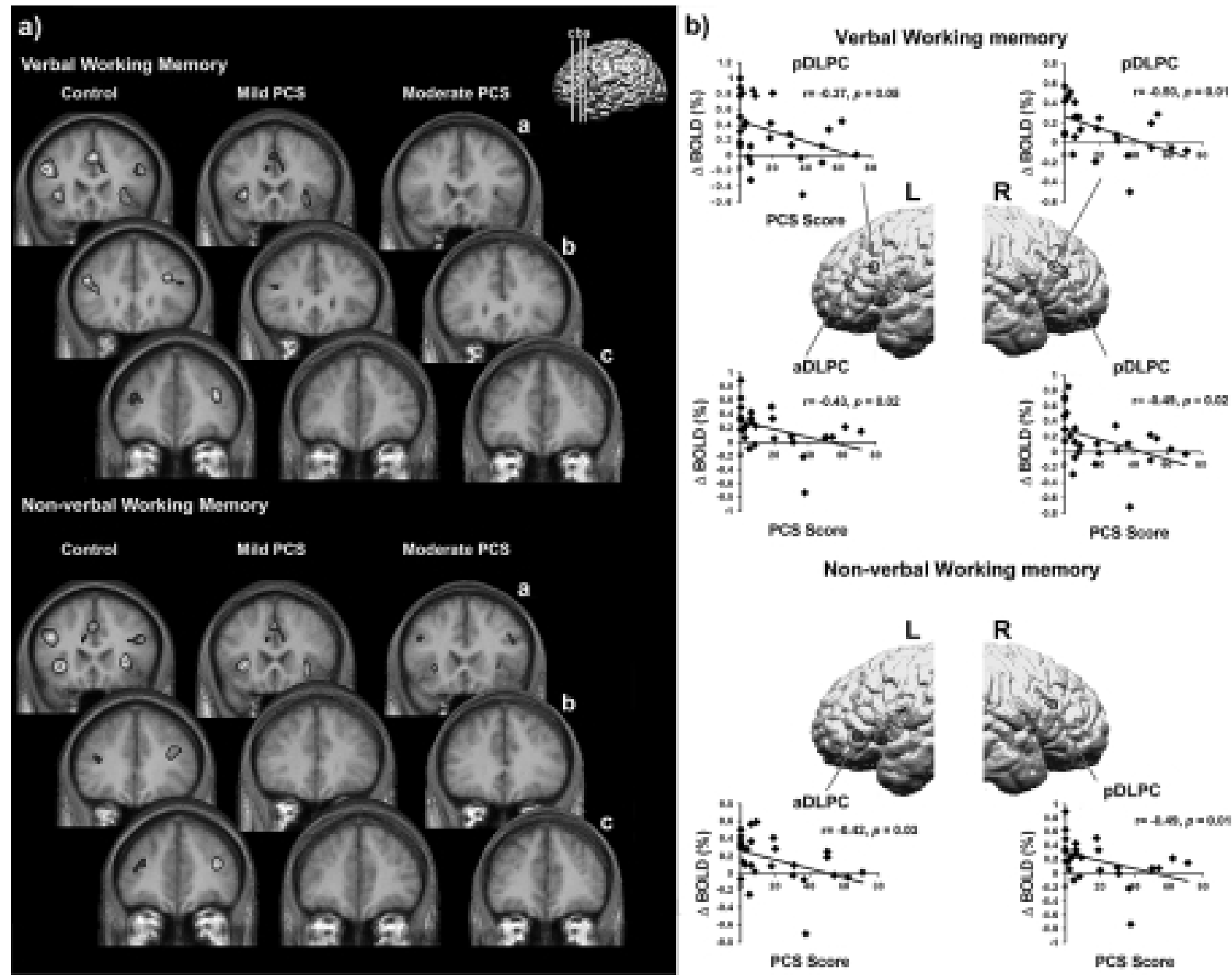

Non-verbal Working memory

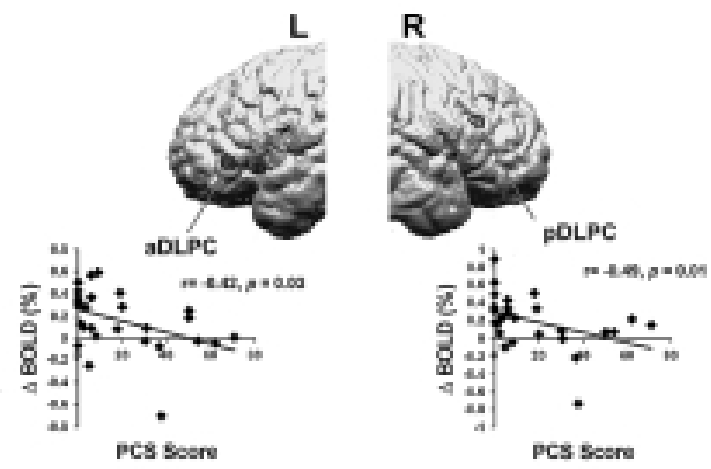

Fig. 2. a) Group average activation maps for the verbal and non-verbal working memory tasks, superimposed on each group's average MRI. Note: task-related brain activities are reduced in athletes with concussion and persisting post-concussive symptoms. b) PCS score and BOLD change correlation. An inverse relationship between PCS score and \% BOLD signal change is observed in the prefrontal regions of interest. (aDLPC $=$ anterior dorsolateral prefrontal cortex; $\mathrm{pDLPC}=$ posterior dorsolateral prefrontal cortex). Adapted from [38].

less of whether they experienced loss of consciousness at the time of the injury (Fig. 1). More specifically, the performances of the athletes varied from chance to normal but they had weaker and fewer task-related activations than the controls. In some cases, particularly in those that had multiple concussions, there was a lack of task-related activation and the activation peaks were mainly located within the posterior prefrontal region. The authors examined percent BOLD changes in the regions of interest of single individuals and compared them to those of the control subjects. The results show a greater increase in DLPC bilaterally for control subjects and an athlete who performed well (the only asymptomatic concussed athlete), while \% BOLD change for an athlete who performed poorly was comparatively low. In that study, an athlete with multiple concussions was tested twice: six months following his last concussion and three months later. Consistent with an improvement in his subjective complaints and dissipation of PCS, the results showed an increase in his behavioral score on the visual version of the task and an augmentation of the prefrontal activations associated with the task. These findings suggest that the presence of PCS can be an important, if not the most important, indicator for the diagnosis and prognosis of concussive injury, and that it can have significant implications in establishing a concussion grading scale. The results also suggest that working memory tasks as well as fMRI imaging are useful in identifying an underlying fronto-cortical dysfunction following concussion.

While earlier concussion guidelines had placed emphasis on loss of consciousness as a primary diagnos- 


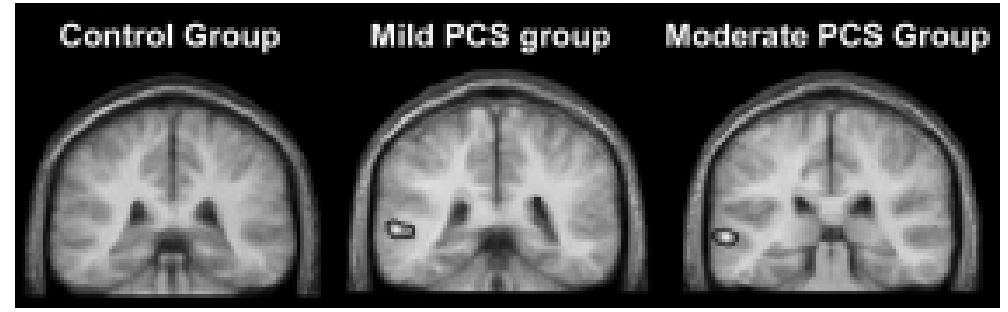

Fig. 3. Additional brain activations in the temporal regions of both groups with PCS, not seen in the controls. Adapted from [38].

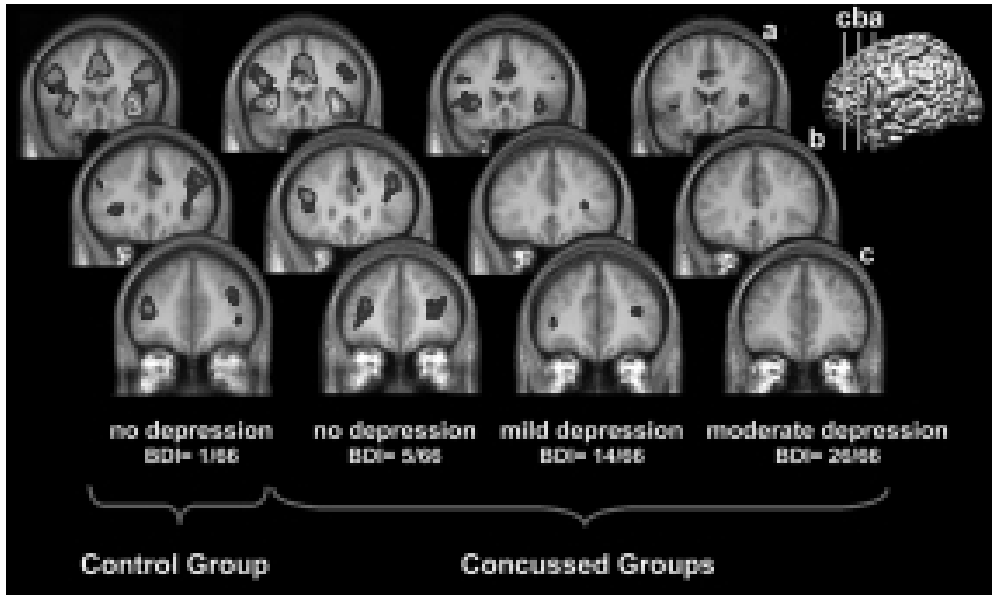

Fig. 4. fMRI results showing different task-related brain activities in control subjects and concussed athletes with symptoms of depression. Note: $\mathrm{a}, \mathrm{b}, \mathrm{c}=$ slice level in the prefrontal cortex.

tic indicator, these have been abandoned in favor of a multifactorial approach, in which PCS are key markers in the assessment of cerebral concussion and in making return-to-play decisions $[6,12,14,33,43]$.

\subsection{Revised definition of concussion, PCS and fMRI}

The Concussion in Sport Group (CISG) [2-4] recommended that combined measures of recovery should be utilized to assess injury severity (and/or prognosis) and guide return-to-play decisions rather than previous grading systems. In the case of concussed athletes, there is a need for tools with which to measure severity of concussive injury as well as effects of intervention or management. Players who have had a concussion are at a greater risk of impaired playing performance, further injury, and sustaining another impact before recovery from the initial trauma [7,23,29,42]. In addition, PCS may become persistent and refractory to treatment $[1,23]$. These findings have important implications with respect to grading of concussion, management and return-to-play guidelines [14,21,24, 27,31]. Neurological examinations and mental status evaluation offer only gross measurements and may not detect subtle findings that are often present, which may account for missed diagnoses and premature return-toplay decisions. Consequently, new approaches to evaluating injury-severity emphasize the lower grade injuries $[6,33]$, as they are often unrecognized, and stress the importance of PCS $[9,18]$.

Although this approach is helpful in facilitating clinical judgment and patient management, concern has been raised as to the reliability of self-reported symptoms. To address this issue, a study [9] was carried out in our laboratory to examine the relationship between self-reported PCS and results on a computerized cognitive test battery and fMRI activation studies in a group of concussed athletes with persisting PCS ("complex concussion") [43]. The primary objective was to evaluate the use of the PCS score as a diagnostic indicator in complex concussion and to investigate whether the severity of self-rated symptoms reflects the degree of ongoing behavioral and functional changes after a cerebral concussion. The results showed that, as a group, athletes with a PCS score greater than 21 (moderate severity) showed significantly slower response times 
a)
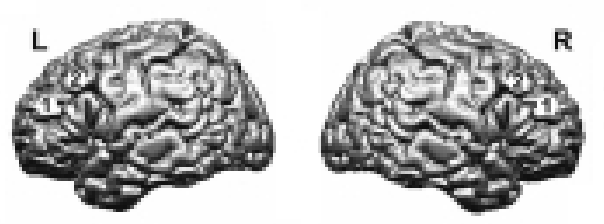

QMid-DLPC (mDLPC)

QPosharioe DLPC (pDLPC)

b)
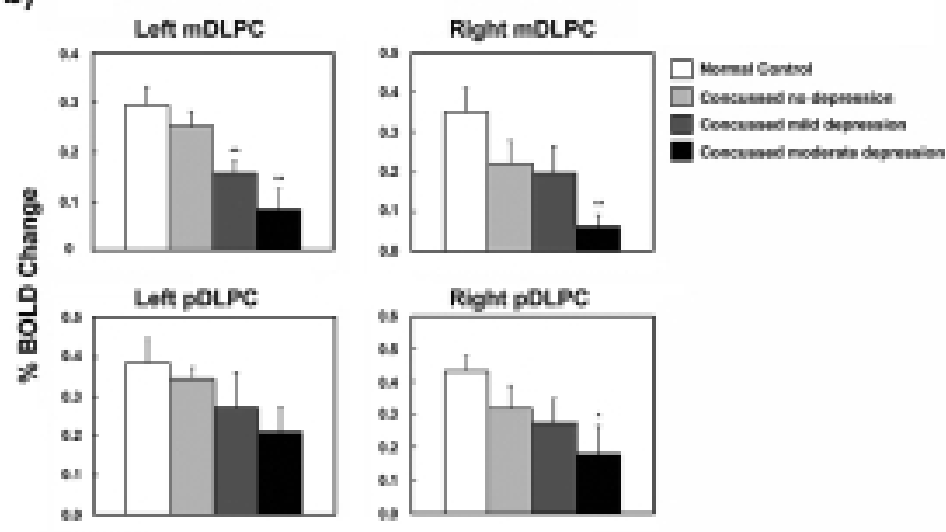

c)

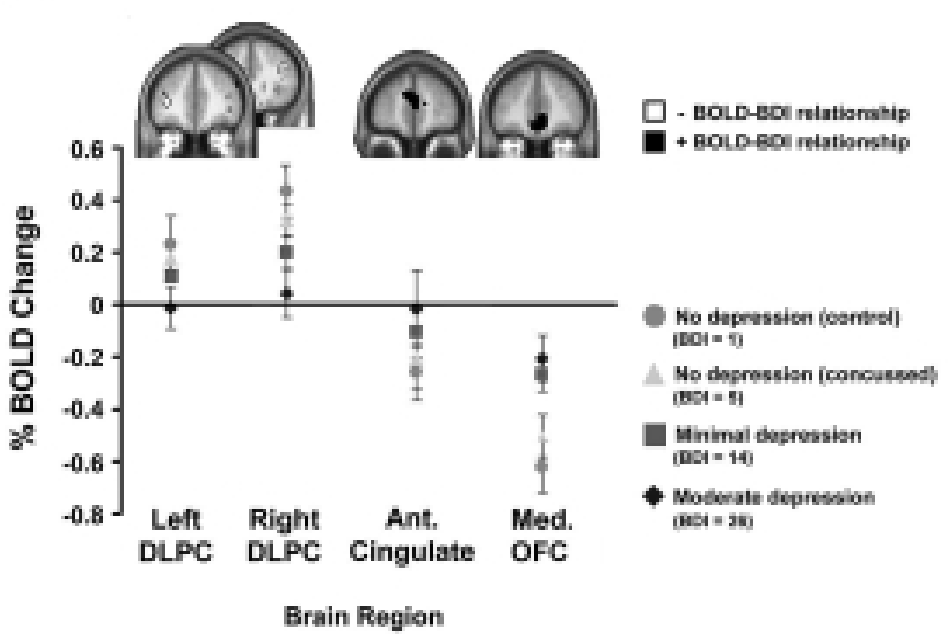

Fig. 5. Quantitative analyses control vs. depressed athletes with concussion. a) Areas where significant task-related peaks were identified and chosen for quantitative analyses; b) Percent BOLD change from baseline condition in each region of interest; c) Relationship between depression scores and \% BOLD signal changes: the higher the depression score, the lower the \% BOLD signal changes in the right and left DLPC; the higher the depression score, the lower the decrease in negative \% BOLD signal changes in the anterior cingulate (ACC) and medial orbitofrontal cortices (Med. OFC)

than the control group on the matching and one-back tasks from the computerized cognitive battery. In addition, their functional MRI results displayed an almost complete absence of activation in the prefrontal regions of interest when performing a working memory task (Fig. 2a). This lack of cerebral activity in the frontal region was seen for both verbal and non-verbal tasks.
In contrast, athletes with a PCS score ranging from 6-21 (mild severity) performed as well as the normal control group on the cognitive test battery. When tested with fMRI, they nevertheless showed reduced fMRI signals in the prefrontal regions of interest compared with the normal control subjects, although the foci were significantly greater than those shown by the moderate 
PCS group. Regression analyses were performed on the PCS scores and BOLD signal changes to examine the relationship between the self-reported PCS and the fMRI activation pattern. The result confirmed a negative relationship between BOLD signal change and the severity rating of PCS (Fig. 2b).

Furthermore, additional brain activations in the temporal regions of both groups, not seen in the controls, were observed (Fig. 3). Interestingly, the authors have noted similar 'atypical' activation foci in a different group of athletes, and they attributed them to the use of compensatory mechanisms through recruitment of extra neuronal resources [12]. Taken together, these results support the ability of the self-rated PCS score to suggest different degrees of behavioral and functional deficits $[8,9,11]$.

To our knowledge, this study was the first to validate with functional neuroimaging and cognitive testing the self-rated PCS scale and to confirm that there is a physiological correlate to PCS. It also showed that the presence of self-reported symptoms is a scientifically valid measure of injury severity and strengthened the clinical validity of the PCS scale as a diagnostic tool. Further, it highlighted the potential of fMRI, in conjunction with the working memory task, to detect abnormalities among symptomatic concussed athletes who had normal structural imaging results $[8,9,11]$.

\subsection{Depression following concussion and fMRI}

In addition to somatic symptoms such as headaches and fatigue and cognitive deficits (e.g. memory, concentration, planning and organization), PCS include psychiatric complications, such as anxiety, irritability and depression. Most studies on sport concussion have focused on the somatic and cognitive aspects, while the psychiatric dimension of PCS remains largely unexplored. This may be particularly relevant in sport concussion [26] as depression has readily been attributed to the loss of position on the team, lack of team support environment, the lack of defined timeline to recovery and the fact that this injury is "invisible", raising issues of compliance or malingering. An acknowledgement of the importance of affective symptoms with concussion injury and recovery was raised at the most recent International Concussion Symposium [43]. In addition, the anecdotal success of treatment with antidepressants in isolated sport concussion cases raises awareness of the contribution of mood symptoms to this pathology.

To understand better the underlying neural mechanisms of mood disturbance, particularly depression ex- pressed by athletes following a cerebral concussion, a fMRI study was carried out in our laboratory to compare neural responses associated with a working memory task between athletes with concussion who complained of depression, athletes with concussion who did not complain of depression and non-injured control athletes $[10,11]$. Athletes in the three groups filled out the Beck Depression Inventory (BDI-II) and underwent an fMRI session during which they performed our externally ordered working-memory task known to engage prefrontal cortex $[17,20]$. The non-injured control group as well as the athletes with concussion who did not report depression (BDI-II score less than 10) exhibited the expected increase in fMRI signal in the DLPC and ventrolateral prefrontal (VLPC) regions, consistent with our previous findings (Fig. 4). Those athletes with concussion who complained of depression were divided into two groups according to the severity of their depressive symptomatology: mild (BDI-II score between 10 and 19) and moderate (BDI-II Score between 20 and 29). No athlete complained of severe symptoms. Results showed that those athletes with concussion and mild symptoms of depression have attenuated fMRI signals in the DLPC while the reduction in activation is even more pronounced in those with moderate symptoms of depression (Fig. 4).

Volume-of-interest (VOI) analyses were carried out to quantify the observed differences in neural activities. Four VOIs were identified in the prefrontal cortices (two in each hemisphere). The corresponding mean percent BOLD signal change from the baseline to working memory condition was then calculated. Athletes with mild depression showed significantly less BOLD signal increases than the control group in the left anterior DLPC while the moderately depressed athletes showed further reduction in BOLD signal increases in the right and left anterior DLPC, and right posterior DLPC (Fig. 5a-b).

To examine further the relationship between brain activations and the severity of depressive symptoms, scores on the BDI-II were used as a covariate to identify brain regions where changes in BOLD signals are modulated by the scores on the depression scale. The results showed that the magnitude of fMRI BOLD signals in both right and left DLPC was negatively correlated with the severity of depressive symptoms as assessed with the BDI-II (Fig. 5c). It was also noted that the BOLD signals in the anterior cingulate (ACC) and medial orbitofrontal cortices (mOFC) were positively correlated with BDI-II scores (Fig. 5c). A crucial difference in the ACC and OFC between the two groups 


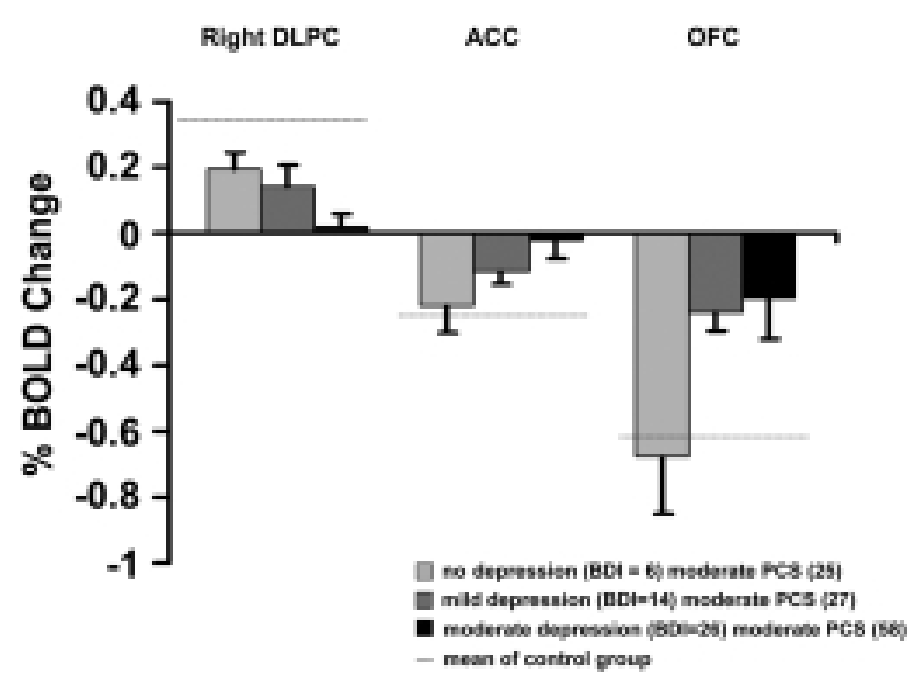

Fig. 6. Relationship between post-concussive symptoms and depression. BOLD signal attenuation in the right DLPC occurred in both depressed and non-depressed concussed athletes. Athletes with PCS and depression showed more signal reduction than athletes with PCS alone. Athletes with depression showed smaller negative BOLD signals in ACC and mOFC. This pattern was not seen in non-depressed concussed athletes with PCS.

with high PCS scores with and without depression was observed, however. In these cerebral areas, athletes with high PCS scores but not depression showed similar negative BOLD signal changes as the control group. In contrast, these negative BOLD responses were significantly attenuated in those athletes with high PCS scores and symptoms of depression.

This difference can be illustrated further by contrasting the BOLD responses of this group with those reporting mild depressive symptoms. Both groups had similar PCS scores (25 versus 27) and showed reduced activations in the right DLPC when compared to the control subjects (Fig. 6). However, only those with depression had significantly less task-related BOLD decreases in the ACC and $\mathrm{mOFC}$, and they showed greater signal reduction in the right DLPC compared with the non-depressed but high PCS score group. Thus, while the reduced brain activations in the right DLPC were likely due to the combination of the PCS and depressive symptoms, the changes in ACC and mOFC appear primarily related to the presence of depressive symptoms.

One explanation for the decrease in negative BOLD signals is an overall increase in neural activities in the ACC and OFC of the depressed athletes. Elevated neural activities in these regions have been associated with experimentally induced anxiety and sadness in normal subjects and patients with major depressive disorder (MDD) [36]. PET studies on major depression have also reported increased metabolism in the ACC, reversible with antidepressant medication [16]. In a study using fMRI, Rose et al. [52] reported that patients with
MDD had relatively higher fMRI signals in the medial OFC and ACC when performing an $n$-back working memory task than normal control subjects. Although none of our athletes with symptoms of depression were diagnosed with MDD, our results are strikingly similar to functional neuroimaging findings in major depression and suggest that depression following head trauma may share the same underlying neural mechanism as MDD.

Studies that examined outcomes following traumatic brain injury have reported a prevalence of depression a few years after the injury $[15,28,32,52,53]$. The question arises as to whether the symptoms of depression represent an emotional reaction to the trauma and to the ongoing PCS, or whether there is an organic basis to them. Recently Jorge et al. [28] reported that depression following head trauma was equally frequent among mild, moderate and severe cases one year postinjury. In addition to worse functional outcome, head injury patients with depression also showed significantly reduced gray matter volumes in the left prefrontal cortex. Levin et al. [34,35] found that mTBI patients with documented CT scan lesions were at greater risk of developing major depressive episodes three months post-injury. These findings, together with our results, point to an organic basis to the depressive symptomatology following brain trauma. Our data also indicate that the presence of depressive symptoms is associated with a greater reduction in cerebral activity in the DLPC than with PCS alone and are consistent with reports 


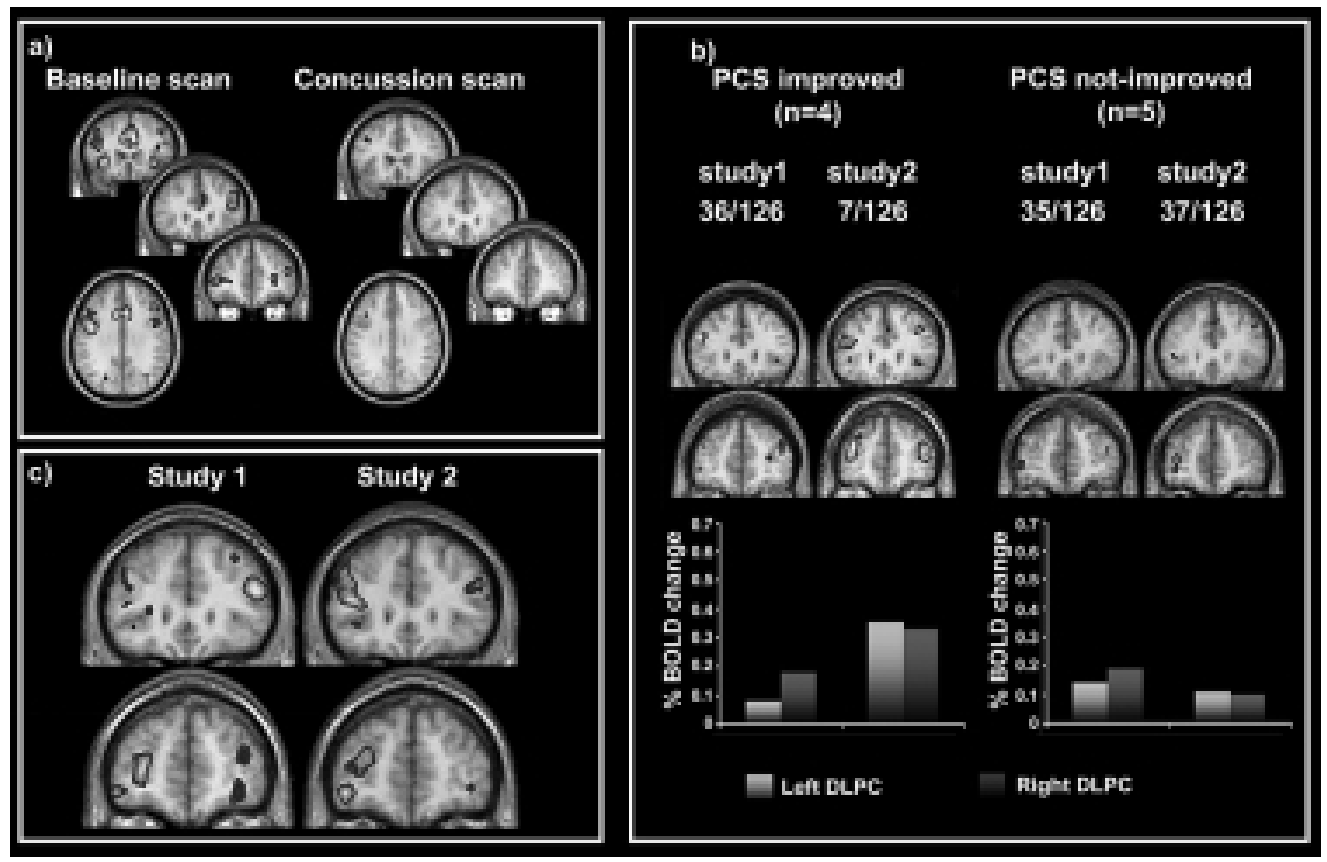

Fig. 7. Serial fMRI studies. a) Comparison of brain activations associated with the working memory tasks at baseline (PCS score $=4$ ) and after concussion ( $<72$ hrs; PCS score $=27)$ in 8 subjects. Note reduced activations; b) Serial fMRI results from concussed athletes with symptom resolution and with no symptom resolution. Note: task-related brain activities in the frontal region are clearly observed only in athletes with symptom resolution; c) Serial fMRI results from 4 healthy normal athletes. The results demonstrate consistent task-related brain activities in the prefrontal region, indicating good test-retest reliability.

of greater disability and poorer outcome in depressed head trauma patients.

\subsection{Serial fMRI in concussion: The McGill Concussion Study (ongoing)}

A major advantage of the fMRI technique is its noninvasive nature which allows serial testing in the same subject within a short time span. This has great implication in a clinical setting since it permits monitoring of recovery in concussed individuals. In addition, intrasubject comparisons are more likely to be sensitive to change than intersubject comparisons and use of normative data. Since 2003, the present authors have built with support from the Canadian Institute of Health Research, a solid database of 83 McGill varsity hockey and football athletes who were free of neurological and/or psychiatric disorders, and who did not have a concussion at least in the preceding 12 months. These players still have at least two-three years of playing time such that they are being followed and new additions to the teams can also be recruited. The databank includes: a) baseline PCS assessment; b) formal neuropsychological testing; c) computerized cognitive testing; d) fMRI studies associated with a work- ing memory task and one that requires navigation and orientation; e) high resolution structural MRI's (DTI, T1, T2 and FLAIR); f) measurements of hippocampal volumes. This procedure allows pre versus post injury comparisons for each individual within the group who may subsequently become concussed.

So far our data indicates that the brain activations (mid-DLPC) associated with the externally ordered working memory tasks for 83 normal athletes were remarkably similar to those reported in our previous studies. We are thus confident of the inter-individual reliability of fMRI. Of the 83 baseline subjects, eight sustained a sport-related concussion and were administered the protocol within 72 hours after the injury. Comparison of the brain activations associated with the working memory tasks at baseline (mean PCS score = 4 ) and after concussion ( $<72 \mathrm{hrs;}$ mean PCS score $=27$ ) in the 8 subjects showed a quasi absence of cerebral activations in the regions of interest (Fig. 7a).

Three athletes with an orthopedic injury were administered the protocol within the same time frame in order to examine the effect of an injury per se. There was almost no change in brain activations associated with the working memory tasks in these athletes with orthopedic injury but not concussion suggesting that the 
reduced brain activity in symptomatic concussed athletes are not simply due to the general effect of having sustained an injury.

Follow-up studies were also carried out following symptom resolution in four athletes with concussion and one orthopedic injury. Task-related brain activities in the frontal region were clearly observed only in those athletes with symptom resolution while the same regions remained relatively silent for those with persistent symptoms (Fig. 7b). The subject with a resolved orthopedic injury showed remarkably similar scans. Finally, the same protocol was re-administered to four uninjured athletes to verify test-retest reliability. The serial fMRI results demonstrate consistent task-related brain activities in the prefrontal region, indicating good testretest reliability (Fig. 7c). Thus, our results show that fMRI is sensitive enough to detect abnormal activation patterns at the individual level and that task-related activations can be consistently and reliably produced $[12$, 26].

\section{Conclusion}

Our aim is to develop the means to evaluate the effects of head trauma as well as to monitor progress in cognitive performance and verify recovery. We believe our studies will provide a new look at brain plasticity in adults. It is important to identify athletes (professional and amateur) at high risk for persistent PCS because these individuals will benefit from counselling with regard to return to play, retirement from sport, increased vulnerability, and concussion rehabilitation and protection issues. Repeated concussion can have serious effects on cognition. This is known. However, it is not clear what the signs are that indicate major upcoming cognitive problems. Given the opportunity to reassess athletes with mTBI with neuropsychological evaluation, structural imaging and fMRI, we may be able to establish patterns of performance both in neuropsychological test profile, structural MRI and fMRI indices suggesting that any further mTBI in a given athlete could prove serious. If such signs could be established, they could impact the practice of sports medicine where both the athletes and their teams may underestimate the potential cumulative effect of repeated mTBI. Such signs and criteria can only be established if one can follow athletes longitudinally with detailed evaluation (clinical, neuropsychological, structural and fMRI scanning). Ultimately, we hope to extrapolate our results to other populations suffering
mTBI (motor vehicle accidents, falls, older adults, and children; e.g. see Fig. 6 for \% BOLD change in regions of interest expected for normals) because the economic cost to society (e.g. lost working hours, reduced quality of life) is so great and needs to be reduced.

\section{References}

[1] S.D. Anderson, Post-concussional disorder: common result of head injury, Can J Diagn 12 (1995), 77-86.

[2] M. Aubry et al., Summary and agreement statement of the $1^{\text {st }}$ International Conference on Concussion in Sport, Vienna 2001, British Journal of Sport Medicine 36 (2002), 6-10.

[3] M. Aubry et al., Summary and agreement statement of the $1^{\text {st }}$ International Conference on Concussion in Sport, Vienna 2001, Clinical Journal of Sport Medicine 12 (2002), 6-1.

[4] M. Aubry et al., Summary and agreement statement of the $1^{\text {st }}$ International Conference on Concussion in Sport, Vienna 2001, Physician \& Sport Medicine 30 (2002), 57-63.

[5] R.C. Cantu, Concussion severity should not be determined until all postconcussion symptoms have abated, Lancet Neurology 3 (2004), 437-438.

[6] R.C. Cantu, Posttraumatic retrograde and anterograde amnesia: Pathophysiology and implications in grading and safe return to play, J Athletic Training 36 (2001), 244-248.

[7] R.C. Cantu, Return to play guidelines after a head injury, Clin J Sport Med 17 (1998), 45-60.

[8] J.K. Chen, K.M. Johnston, A. Collie, P. McCrory and A. Ptito, Association between symptom severity, CogSport tests results and fMRI activation in symptomatic concussed athletes, Clinical Journal of Sport Medicine 14 (2004), 379.

[9] J.K. Chen, K.M. Johnston, A. Collie, P. McCrory and A. Ptito, A validation of the post concussion symptom scale in the assessment of complex concussion using cognitive testing and functional MRI, J Neurol Neurosurg Psychiatry (2007), in press.

[10] J.K. Chen, K.M. Johnston and A. Ptito, Severity of depression correlates with fMRI activation in the prefrontal cortex following sport-related concussion. Abstract No. 541 W-AM. 2006 Abstract Viewer, Florence, Italy. Human Brain Mapping, 2006. Online.

[11] J.K. Chen, K.M. Johnston, M. Petrides and A. Ptito, Neural substrates of symptoms of depression following concussion in male athletes with persisting post-concussion symptoms, Archives of General Psychiatry, in press.

[12] J.K. Chen, K.M. Johnston, S. Frey, M. Petrides, K. Worsley and A. Ptito, Functional abnormalities in symptomatic concussed athletes: an fMRI study, Neuroimage 22 (2004), 6882.

[13] M.W. Collins et al., Relationship between concussion and neuropsychological performance in college football players, JAMA 282 (1999), 964-970.

[14] Committee CASMC. CASM Guidelines for assessment and management of sport-related concussion, Clin J Sport Med $\mathbf{1 0}$ (2000), 209-211.

[15] S. Deb, I. Lyons and C. Koutzoukis, Neuropsychiatric sequelae one year after a minor head injury, J Neurol Neurosurg Psychiatry 65 (1998), 899-902.

[16] W.C. Drevets, Prefrontal cortical-amygdalar metabolism in major depression, Ann N Y Acad Sci, 877 (1999), 614-637. 
[17] J. Duncan and A.M. Owen, Common regions of the human frontal lobe recruited by diverse cognitive demands, Trends Neuroscience 23 (2000), 475-483.

[18] F. Dupuis, K.M. Johnston, M. Lavoie, F. Lepore and M. Lassonde, Concussions in athletes produce brain dysfunction as revealed by event-related potentials, NeuroReport 11 (2000), 4087-4092.

[19] M. Gaetz, D. Goodman and H. Weinberg, Electrophysiological evidence for the cumulative effects of concussion, Brain Injury 14 (2000), 1077-1088.

[20] R.L. Gould, R.G. Brown, A.M. Owen, D.H. Ffytche and R.J. Howard, fMRI BOLD response to increasing task difficulty during successful paired associates learning, Neuroimage $\mathbf{2 0}$ (2003), 1006-1019.

[21] K.M. Guskiewicz, B.L. Riemann, D.H. Perrin and L.M. Nashner, Alternative approaches to the assessment of mild head injury in athletes, Med Sci Sports Exerc 29 (1997), S213-221.

[22] K.M. Guskiewicz, S.W. Marshall, S.P. Broglio, R.C. Cantu and D.T. Kirkendall, No evidence of impaired neurocognitive performance in collegiate soccer players, Am J Sports Med 30 (2002), 157-162.

[23] H. Heinz, T. Munte, W. Gobiet, H. Niemann and R. Ruff, Parallel and serial visual search after closed head injury: Electrophysiological evidence for perceptual dysfunctions, $\mathrm{Neu}$ ropsychol 30 (1992), 495-514.

[24] C.R. Honey, Brain injury in ice hockey, Clin J Sport Med 8 (1998), 43-46.

[25] K.J. Jantzen, B. Anderson, F.L. Steinberg and J.A.S. Kelso, A prospective functional MR imaging study of mild traumatic brain injury in college football players, Am J Neuroradiol 25 (2004), 738-745.

[26] K.M. Johnston et al., Current Concepts in Concussion Rehabilitation, Current Sports Medicine Reports 3 (2004), 316323.

[27] K.M. Johnston, P. McCrory, N. Mohtadi and W. Meeuwisse, Evidence based review of sport-related concussion - clinical science, Clin J Sport Med 11 (2001), 150-160.

[28] R.E. Jorge et al., Major depression following traumatic brain injury, Arch Gen Psychiatry 61 (2004), 42-50.

[29] P. Karzmark, K. Hall and J. Englander, Late-onset postconcussion symptoms after mild brain injury: the role of premorbid, injury-related, environmental, and personality, Brain Injury 9 (1995), 21-26.

[30] J.P. Kelly and J.H. Rosenberg, Diagnosis and management of concussion in sports, Neurology 48 (1997), 575-580.

[31] J.P. Kelly, J.S. Nichols, C.M. Filley, K.O Lillehei, D. Rubinstein and B.K. Kleinschmidt-DeMasters, Concussion in sports, JAMA 266 (1991), 2867-2869.

[32] J.S. Kreutzer, R.T. Seel and E. Gourley, The prevalence and symptom rates of depression after traumatic brain injury: a comprehensive examination. Brain Injury 15 (2001), 563-576.

[33] S. Leclerc, M. Lassonde, S. Delaney, V.J. Lacroix and K. Johnston, Recommendations for grading of concussion in athletes, Sports Medicine 31 (2001), 629-636.

[34] H.S. Levin et al., Depression and posttraumatic stress disorder at three months after mild to moderate traumatic brain injury, J Clin Exp Neuropsychol 23 (2001), 754-769.

[35] H.S. Levin et al., Predicting depression following mild traumatic brain injury, Arch Gen Psychiatry 62 (2005), 523-528.

[36] M. Liotti et al., Differential limbic-cortical correlates of sadness and anxiety in healthy subjects: implications for affective disorders, Biol Psychiatry 48 (2000), 30-42.
[37] J.T. Matser, A.G. Kessels, B.D. Jordan, M.D. Lezak and J. Troost, Chronic traumatic brain injury in professional soccer players, Neurology 51 (1998), 791-796.

[38] J.T. Matser, A.G. Kessels, M.D. Lezak and J. Troost, A doseresponse relation of headers and concussions with cognitive impairment in professional soccer players, J Clin Exp Neuropsychol 23 (2001), 770-774.

[39] J.T. Matser, A.G. Kessels, M.D. Lezak, B.D. Jordan and J. Troost, Neuropsychological impairment in amateur soccer players, JAMA 282 (1999), 971-973.

[40] T.W. McAllister et al., Brain activation during working memory 1 month after mild traumatic brain injury: a functional MRI study, Neurology 53 (1999), 1300-1308.

[41] T.W. McAllister et al., Differential working memory load effects after mild traumatic brain injury, Neuroimage 14 (2001), 1004-1012.

[42] P.R. McCrory and S.F. Berkovic, Second Impact Syndrome, Neurology 50 (1998), 677-683.

[43] P.R. McCrory et al., Summary and Agreement Statement of the $2^{\text {nd }}$ International Conference on Concussion in Sport, Prague 2004, Clin J Sport Med 15 (2005), 48-57.

[44] National Institute of Health Consensus Statement Vol. 16, 1998.

[45] M. Petrides and B. Milner, Deficits on subject-ordered tasks after frontal- and temporal-lobe lesions in man, Neuropsychologia 20 (1982), 249-262.

[46] M. Petrides, Frontal lobes and working memory: evidence from investigations of the effects of cortical excisions in nonhuman primates, in: Handbook of Neuropsychology, (Vol. 9), F. Boller and J. Grafman, eds, Amsterdam: Elsevier, 1994, pp. 59-82.

[47] M. Petrides, Functional organization of the human frontal cortex for mnemonic processing. Evidence from neuroimaging studies, Annals of the New York Academy of Sciences $\mathbf{7 6 9}$ (1995), 85-96.

[48] M. Petrides, Impairments on nonspatial self-ordered and externally ordered working memory tasks after lesions of the mid-dorsal part of the lateral frontal cortex in the monkeys, Journal of Neuroscience 15 (1995), 359-75.

[49] M. Petrides, Monitoring of selections of visual stimuli and the primate frontal cortex, Proceedings of the Royal Society 246 (1991), 293-298.

[50] G.B. Pike and R.D. Hoge, Functional Magnetic Resonance Imaging: Technical Aspects, in: Pain Imaging, Progress in Pain Research and Management, (Vol. 18), K.L. Casey and M.C. Bushnell, eds, IASP Press, Seattle, 2000.

[51] M.H. Rabadi and B.D. Jordan, The cumulative effect of repetitive concussion in sports, Clin J Sports Med 11 (2001), 194 198.

[52] E.J. Rose, E. Simonotto and K.P. Ebmeier, Limbic overactivity in depression during preserved performance on the n-back task, Neuroimage 29 (2006), 203-215.

[53] R.T. Seel et al., Depression after traumatic brain injury: a National Institute on Disability and Rehabilitation Research Model Systems multicenter investigation, Arch Phys Med Rehabil 84 (2003), 177-184.

[54] E.M. Umile, M.E. Sandel, A. Alavi, C.M. Terry and R.C. Plotkin, Dynamic imaging in mild traumatic brain injury: support for the theory of medial temporal vulnerability, Arch Phys Med Rehabil 83 (2002), 1506-1513. 